$5.3 \%$ to $10.6 \%$ in Whites, and from $1.0 \%$ to $2.7 \%$ in Hispanics but only modestly in Blacks from $1.5 \%$ to $2.3 \%$. In logistic regression models predicting participation, the most important predictors of participation were having at least one physical examination (OR 1.46, 95\%-CI 1.34-1.59) in Whites and long-term membership in Hispanics (OR 1.38, 95\%-CI 1.111.69). None of the restrictions significantly predicted participation in Blacks ( $p$ for interaction with race $<0.001$ ). Conclusions: The application of restrictions based on longer membership and regular physical examinations may increase recruitment of non-Hispanic White and Hispanic men for biobank participation.

Keywords: Biobank; Racial disparities

doi:10.3121/cmr.2014.1250.ps2-15

PS2-16:

Racial Disparities in Biobank Participation among Women in Southern California

Corinna Koebnick ${ }^{1}$; Galina Inzhakova ${ }^{1}$; Darios Getahun'; Mary Helen Black $^{1}$; Steven Jacobsen ${ }^{1}$; Miki Nguyen ${ }^{1}$

${ }^{1}$ Kaiser Permanente Southern California

Background/Aims: The aim of this study is to identify predictors of participation in women of different racial groups to optimize future recruitment efforts. Methods: We randomly selected and assessed predictors of participation on Kaiser Permanente Southern California adult female members $(n=107,378)$ between November 2012 and June 2013. Women were asked to provide a saliva sample for a research biobank. Potential predictors of participation were age, long-term membership ( $=5$ years), no significant gaps in health care coverage ( $<3$ month in last 3 years), physical examination within the last 3 years and neighborhood education and income. Results: The overall participation among women was $6.8 \%$. Restricting recruitment to women with $=5$ years of membership, no major gaps in health care coverage, and at least one physical examination within the last 3 years improved participation in Whites (from $7.1 \%$ to 14.3 ) and Hispanics (from $2.5 \%$ to $5.0 \%$ ) but only modestly in Blacks (from $2.0 \%$ to $3.6 \%$ ). In logistic regression models predicting participation, the strongest predictors in Whites and Hispanics were having a physical examination (OR 1.25, 95\%-CI 1.17 1.33 in Whites and 1.45, 1.28-1.65 in Hispanics) and long-term membership (OR 1.19, 95\%-CI 1.12-1.27 in Whites and 1.32, 1.16-1.50 in Hispanics). None of the restrictions significantly predicted participation in Blacks (p for interaction with race $<0.001$ ). Conclusions: Applying restrictions based on regular physical examinations and longer membership may potentially increase participation of non-Hispanic White and Hispanic women in a research biorepository.

Keywords: Biobank; Racial disparities

doi:10.3121/cmr.2014.1250.ps2-16

\section{Health Informatics}

B1-1:

Predictive Modeling to Identify Patients at Risk for Index Hospitalization

Ted Palen ${ }^{1}$; Heather Tavel ${ }^{1}$; James Brill²; Jennifer Bajaj ${ }^{2}$

${ }^{1}$ Kaiser Permanente Colorado; ${ }^{2}$ Colorado Permanente Medical Group

Background/Aims: Preventable hospital readmissions are the focus of many performance metrics. However, little attention has been given to preventing index admissions. The likelihood of hospitalization (LOH) is a statistical model (Verisk Analytics ${ }^{\circledR}$ ) which uses patient historical clinical data and logistic regression to predict the likelihood of admission in the next six months. One of our goals was to test the accuracy of the LOH model to predict hospitalizations. The second goal was to identify possible common intervenable causes for the hospitalization. Methods: We obtained the list of patients with LOH scores in the top 1\% calculated as of July 1, 2012. We then retrospectively identified which of these patients had hospitalizations in the following 6 months. We censored patients who had hospitalizations related to trauma and pregnancy. We performed chart reviews on a subset of the hospitalized patients and classified reasons for the admission into intervenable or non-intervenable causes. Results: Of the patients in the top $1 \%$ of $\mathrm{LOH}$ $(\mathrm{N}=1460$; mean $\mathrm{LOH}=0.47), 412(28.2 \%)$ were hospitalized within 6 months of the $\mathrm{LOH}$ calculation date. The average age and percent female for hospitalized and non-hospitalized patients was 74.1 years, $57.8 \%$ and 73.4 years, $60.0 \%$, respectively (not significant). 324 of the hospitalized patients had no emergency department visits in the prior 6 months. Fifty percent of hospitalizations occurred within 33 days of the $\mathrm{LOH}$ calculation date. We performed chart reviews on $134(32.5 \%)$ of the hospitalized patients. Twenty-eight patients $(20.9 \%)$ were classified with intervenable causes for the admission, $19(67.9 \%)$ of which were related to system issues such as inadequate follow-up after procedures or medication adjustments. Conclusions: Identifying patients with intervenable causes for hospitalizations may significantly decrease unnecessary admissions. Risking algorithms may have utility in developing strategies to identify these patients. Risk of Hospitalization ( $\mathrm{RHO} 2)$ uses near real-time clinical data from the electronic medical to calculate risk scores. Future studies will compare the performance characteristics of $\mathrm{LOH}$ to $\mathrm{RHO} 2$ to identify overlapping cohorts of patients. If $\mathrm{RHO} 2$ performs as well as $\mathrm{LOH}$, we will use the near-real time clinical data in $\mathrm{RHO} 2$ to flag patients with intervenable causes of hospitalization at the point-of-care.

Keywords: Predictive modeling; Risk profiling

doi:10.3121/cmr.2014.1250.b1-1

B1-3:

Improving Surgical Case Duration Accuracy with Advanced Predictive Modeling

Ronald Dravenstott ${ }^{1}$; Eric Reich ${ }^{1} ;$ Steven Strongwater ${ }^{1} ;$ Priyantha Devapriya $^{1}$

${ }^{1}$ Geisinger Health System Clinical Innovations

Background/Aims: The Operating Room (OR) is a large source of revenue and one of the most costly departments in a hospital. Scheduling surgeries into an OR is complicated by the inherent uncertainty associated with each surgery. The case length of a surgery at Geisinger is predicted using a moving average of the 10 previous procedures performed by a given surgeon. A process capability analysis was performed to gauge the ability of each surgical procedure to be within \pm 15 minutes of scheduled time. This analysis demonstrated a low process capability across all surgeries. This research aims to create a process to better predict the surgical case length by leveraging the Electronic Health Records, which can enable more efficient scheduling and use of the ORs. Methods: Based on a literature review and the results of an internally conducted survey of OR staff, a dataset was constructed with 135 predictors. A test dataset was randomly separated from the training dataset for validation. Predictive models were developed using Stepwise Linear Regression (LR) and Artificial Neural Networks (ANNs). Multilayer Perceptron ANNs with 2 hidden layers using a sigmoid transfer function and Delta Bar Delta learning algorithm tended to perform the best. The final model contains the 39 most sensitive predictor variables from the ANN model and the LR model. Results: In all cases, the predictive models significantly improved the case duration accuracy. The ANN models outperformed the LR models on 3 of the 5 high-volume procedures. The greatest improvement over the baseline occurred for the ANN model for Arthroplasty Total Hip, where case duration accuracy improved from $32.1 \%$ ( 80 of 249 test cases) to $59.0 \%$ (147 of 249 test cases) for an improvement of $83.7 \%$ (improving to $59.0 \%$ from $32.1 \%$ ). Conclusions: The ANN and LR models can be used to significantly enhance the predictability of surgical schedules. Even with the significantly enhanced predictability of surgical case lengths, the 5 investigated high-volume surgeries are still not necessarily process capable with respect to \pm 15 minute specification limit.

Keywords: Predictive modeling; Operating room

doi:10.3121/cmr.2014.1250.b1-3

B1-4:

eCare for Moods (TM), A Patient-centered, Web-delivered Selfmanagement and Care Management Program for Recurrent Depression: Results from Randomized Trial

Enid Hunkeler ${ }^{1}$; William Hargreaves ${ }^{2}$; Bruce Fireman ${ }^{1}$; Yvonne Porterfield ${ }^{1}$; Gregory $\mathrm{Simon}^{3}$

${ }^{1}$ Kaiser Permanente Northern California; ${ }^{2}$ University of California San Francisco; ${ }^{3}$ Group Health Research Institute 
Background/Aims: We assessed the effectiveness of eCare for Moods ${ }^{\mathrm{TM}}$ (patent pending), a patient-centered, highly interactive, web-delivered patient self-management and care management program on patients treated for recurrent or chronic depression in specialty psychiatry through a randomized clinical trial with two-year follow-up. Methods: Patients with recurrent or chronic depression were randomly assigned to eCare $(\mathrm{N}=51)$ or usual specialty mental health care $(\mathrm{N}=52)$. The 12-month eCare program provided patients with individualized self-monitoring, tailored patient education and training in depression self-management including relapse prevention. eCare was integrated with participants' ongoing depression care, linked to their electronic medical records. It provided clinicians with panel management and clinical decision support. Participants were interviewed at baseline and $6,12,18$, and 24 months after enrollment. Telephone interviewers blind to treatment assignment used a timeline follow-back method to estimate depression severity on a 6-point scale for each of the 105 study weeks (including the baseline). Differences between groups in weekly severity over two years were examined by generalized estimating equations. Results: Participants in eCare experienced more reduction in depressive symptoms (estimate $=-74$ on the 6-point scale over two years; $95 \%$ confidence interval $[\mathrm{CI}]=-1.38$ to $-.09, P=.025)$ and were less often depressed $(-.24$ over two years; $\mathrm{CI}=.46$ to $-.03, P=.026)$. At 24 months, $43 \%$ of eCare and $30 \%$ of usual-care participants were depression free; the number needed to treat to attain one additional depression-free participant was 8. eCare participants had other favorable outcomes: improved general mental health $(P=.002)$, greater satisfaction with specialty care $(P=.003)$ and with learning new coping skills $(P<.001)$, and more confidence in managing depression $(P=$ .006). Conclusions: Patient-centered, web-delivered care management improves outcomes in patients treated for recurrent and chronic depression. Keywords: Internet-delivered care; Depression

doi:10.3121/cmr.2014.1250.b1-4

C3-1:

Footprints in the Sand: Tracking Physician Work Efforts in Primary Care Using Access Logs in an Electronic Health Record

Ming Tai-Seale ${ }^{1}$; Caroline Wilson ${ }^{1}$; Cliff Olson ${ }^{2}$; Meg Durbin ${ }^{1}$; Criss Morikawa ${ }^{1}$; Hal Luft ${ }^{1}$

${ }^{1}$ Palo Alto Medical Foundation Research Institute; ${ }^{2}$ Palo Alto Medical Foundation for Healthcare, Research and Education

Background/Aims: Using EpicCare Electronic Health Record (EHR) data in a large multispecialty ambulatory delivery system, we explore a unique opportunity in which existing EHR data may offer clues on how clinicians use time, a scarce yet critical resource in health services delivery. Traditional means of studying physician time use during clinical encounters (e.g., direct observation) are costly and ignore pre-service and post-service work of physicians' services. The EpicCare EHR offers an alternative, unobtrusive portal to study time use through analysis of access logs. Methods: We used EHR access log data for one month in 2013 from 49 physicians in two primary care departments who cared for 22,174 patients in a large multispecialty ambulatory delivery system. Over 3 million EHR transactions are examined to explore individual physicians' style of time use on different tasks, as reflected by the access log. In-depth key informant interviews are used to complement the access log data on how physicians use the EHR and the activities that are more or less likely to be captured by the access log. Results: About $43.7 \%$ of physicians' total time for the month involved in-person face-to-face visits, 33.8\% involved pre and post visit time, $11.4 \%$ telephone calls, $5.6 \%$ secure messaging to patients, $2.6 \%$ prescription refills, and $1.6 \%$ on orders for labs, medications or referrals. The earliest EHR access in the office occurred at 12:00 am and the latest logging out time in the office was at 11:59 am the following day. For each patient visit, an average of 16.7 minutes was logged in the exam room and 7.9 minutes logged outside of the exam room. Conclusions: The access log is a valuable tool for studying physician work efforts. Our findings highlight the significant amount of time clinicians spend outside of office visits. Unless there is a fixed ratio of in-office to total time, visit-centric FFS payment may undercompensate the significant efforts outside of visits. As "desktop medicine" (e.g., via phone, messaging) increases in the age of the Internet, smart phones, and EHRs, reforming provider payment mechanisms to account for work outside of office visits is warranted.

Keywords: Physician work efforts; EHR access log

doi:10.3121/cmr.2014.1250.c3-1

C3-2:

All-In-One: How Group Health Organizes Clinical Text from Clarity for Research

Scott Halgrim ${ }^{1}$; David Carrell ${ }^{1}$; Diem-Thy Tran $^{1}$

${ }^{1}$ Group Health Research Institute

Background/Aims: Group Health Cooperative runs Epic as its Electronic Medical Record. The clinical text stored in Clarity, Epic's relational reporting database, is valuable to research at Group Health Research Institute (GHRI). However, due to a number of factors, GHRI's access to this data was limited. These factors included: 1) a limited window during the day allotted to GHRI due to higher priority reports on Group Health's care delivery side, 2) Clarity splitting text notes into lines of about 5,000 characters, and 3) restrictions on managing the database itself, like adding a full-text index. We sought to make the clinical text more valuable for research by making it available at all times, combining all the content of a note into one record, and allowing for more database management options. Methods: We have developed a nightly Python process that moves clinical text from four Clarity tables into one fulltext-indexed table on our own server. In addition, we store metadata about each note--including note type, encounter date, department, and provider--in a parallel table. Results: This conversion process, begun in 2010, converts about 60,000 notes per night and has converted every extant note in Group Health's Clarity database for a total of 123 million notes as of October 2013. The notes' availability has sped development of sophisticated NLP algorithms in the years since its inception. Another benefit is nightly automated status e-mails sent to the developers. When there was a recent import of several years of historical notes from legacy systems into Epic, GHRI knew immediately that a greater history of notes was available for research. Conclusions: The text store at GHRI has strengthened research and grant submissions. Due to Clarity's consistent data model and large footprint throughout the nation's medical community, the solution should be easily transferable to other sites wishing to realize the same advantages. The solution is amenable to enhancement as needs arise for more metadata or for clinical text from other parts of Clarity. Remaining challenges are tracking changes to notes in Clarity and improving performance.

Keywords: Natural language processing; Clarity

doi:10.3121/cmr.2014.1250.c3-2

\section{C3-4:}

An Algorithm to Combine Machine Learning and Structured Data to Automate De-identification of Clinical Text

Diem-Thy Tran ${ }^{1}$; Scott Halgrim ${ }^{1}$; David Carrell ${ }^{1}$

${ }^{1}$ Group Health Research Institute

Background/Aims: Clinical text is an important resource for research. To maintain patient privacy when researching this text, we use de-identification. The hiding in plain sight (HIPS) method is promising; it replaces personally identifiable information (PII) with realistic surrogates so any remaining real PII would be hard to distinguish from the fake information. However, there remain some challenges with HIPS, such as overlooked PII. We explored these challenges and hypothesized that we could find more PII by combining structured data with a machine learning algorithm. Methods: The machine learning de-identification software we used, developed by MITRE, is the MITRE Identification Scrubber Toolkit (MIST). Trained chart abstractors annotated Family Practice notes with the following PII types: address, age, date, provider name, email, IP address, consumer number, organization name, other id, phone, patient name, room id, social security number, and URL address. Structured data included in this experiment are patient's address, age, date of birth, email, phone, consumer number, social security number, the visit provider name, visit date, and visit location. We queried this data from Clarity, a relational reporting database for Group Health's electronic health record (EHR) system. Our first test experiment used MIST to train a model on 100 documents then tested on 10 notes. We reviewed the remaining PII and determined if they are available in the structured data. 